\title{
Supply chain security evaluation model and index system based on a $5 \mathrm{G}$ information system
}

\author{
Jingfeng $\mathrm{Zhao}^{1} \cdot$ Yan $\mathrm{Li}^{1}$
}

Received: 24 June 2021 / Accepted: 21 September 2021 / Published online: 29 October 2021

(C) The Author(s), under exclusive licence to Springer-Verlag London Ltd., part of Springer Nature 2021

\begin{abstract}
Currently, the supply chain management model has been widely adopted worldwide. Internationally renowned companies such as IBM and Walmart have made significant achievements in supply chain management and have played a greater role in promoting the growth of their interests. However, while they are enjoying their own interests, they are also facing various risks in the supply chain. From the perspective of systems engineering, this paper uses complex network theory as a research tool to study supply chain networks and systematically study the important issues of supply chain network risks. This paper analyzes the new characteristics of the relationship between enterprises and suppliers in the supply chain and which indicators should be selected as the basis for evaluating suppliers from the perspective of cooperation and development, which more comprehensively reflects the characteristics of the new enterprise-supplier relationship in the supply chain environment. This highlights the evaluation of the supplier's cooperation ability and development ability. Aiming at the problem of supply chain network system identification and integrated evaluation, a supply chain security evaluation model and index system research framework model are proposed, and the intercompany business risk and supply chain in supply chain network evaluation are introduced. As a model for evaluating transition parameters, the study of supply chain risk propagation introduces the theory of dynamic propagation in complex networks, the law of evolution, and the supplier's dynamic supply chain risk model. The experimental results of this paper show that when the global reputation value is 0.5 and the local reputation value is 0.8 and 0.7 , the trust degree changes with the change of the confidence factor; when the value of the confidence factor decreases, the degree of trust converges more and more to the global reputation and vice versa. It can be seen that this model has high feasibility.
\end{abstract}

Keywords 5G Information system · Supply chain security $\cdot$ Evaluation model $\cdot$ Index system

\section{Introduction}

\subsection{Background}

In the supply chain environment, the competition between individual enterprises has become the competition between

Yan Li

b2018120118@stu.ncwu.edu.cn

Jingfeng Zhao

zhaojingfeng@nwu.edu.cn

1 College of Management and Economic, North China University of Water Resources and Electric Power, Zhengzhou 450000, Henan, China the supply chains where the enterprises are located. How to select suppliers scientifically and effectively implement supply chain strategies is a common problem faced by enterprises themselves. With the development of technology and information technology and the advent of the internet era, market-oriented companies are changing rapidly, competition between companies is intensifying, and the frequency of cooperation between companies has greatly increased because it is difficult for a single company to give full play to its advantages. Companies no longer fight alone but rely on the standpoint of supply chain integration to compete in the industry. Especially now, several new business management methods, such as global procurement and sales, outsourcing, inventory reduction, 
lean logistics, and flexible supply chains, are further increasing or expanding the impact of risks. For example, while enjoying the high returns of the global supply chain, companies need to face risks such as social unrest, cultural differences, and economic crises in many countries and regions. Moral hazards and the risk of outsourcing are ignored, and there is a risk of disrupting the operation of a flexible supply chain. With the improvement of the level of supply chain management, the ties between companies are becoming increasingly closer. The problem of an agile supply chain will not only directly damage the business within the chain but also affect the entire supply chain.

\subsection{Significance}

In the context of economic and trade globalization, supply chain structures and interactions have become increasingly complex from traditional chain structures to complex network structures. The behavioral dependence and coupling of operators in the supply chain are also increasing, and the characteristics of synergy and coexistence are becoming increasingly obvious. There are also complex activation relationships among the risk factors of different supply chains. When a specific risk event is triggered, many secondary, derivative, or many other risk events will occur at the same time. Searching for specific links or specific risks in such a complex environment is not only very difficult but also somewhat one-sided. In contrast, studying the overall risk management of the supply chain system from the perspective of the macro-network structure and rules may better solve the objective reality of supply chain operations. The overall operation level of the supply chain system needs to be improved.

\subsection{Related work}

American scholars study the relationship between China's supply chain and the USA. Weber R's study shows an assessment of China's aggressive actions in cyberspace (due to its ambitions, it hopes to rival the USA or replace the USA as a global superpower, and use intellectual property rights), primarily in the form of theft and cyber espionage, have formed unusual commonalities in Congress, which may clear the way for new developments and stricter cyber security requirements to protect industrial supply chains and the overall security of critical infrastructure and US innovation [1]. Although it is in line with expectations in most directions, it does not mention the development factors of China's supply chain due to external environmental factors. In some parts of the forecast, there are errors. To strengthen the stability and efficiency of the national supply chain, Callan B's research shows that the White House issued a presidential executive order to assess and strengthen the US manufacturing and defense industry base and supply chain resilience. The recommendations made by the order were originally scheduled to be completed in April 2018, but the work is progressing smoothly and may be completed soon. Although it is not clear what the scope of this assessment is, it is time for the USA (and other countries) to consider its ability to generate military power [2]. However, the development speed of the supply chain is becoming increasingly faster, and it is obviously inappropriate to predict the future development speed of the supply chain as compared to the previous development speed. Research on the role of the supply chain in certain areas is crucial. Barbosa M W conducted a bibliometric analysis to rigorously examine the evolution of the agri-food supply chain (AFSC) research field from 2008 to 2019. In addition to using different analytical scientific measurement tools (topic mapping, common citations, coauthors, and coverage visualization network), Barbosa also identified commonly used keywords, new and popular research topics, and frequently studied supply chain management (SCM) practice. Frequently used keywords are food supply chain, food waste, sustainability, food safety, SCM, food industry, and food safety. New research topics include contracts, blockchain, Internet of Things, resilience, and short-term food supply chains. This is a topic that needs further research, especially due to the international COVID-19 pandemic and the need for farmers to be closer to consumers [3]. 5G information systems have a place in many fields. Modarress B's research shows that the feedback scheme developed for traditional MIMO systems in LTE is not efficient for massive MIMO systems because the overhead increases almost linearly with the number of antennas. In addition, the massive MIMO codebook is very large, and it is difficult to design using LTE methods. This paper proposes a novel CSI feedback scheme, namely, CSI feedback based on hierarchical multipath information (LMPIF), which can be used in dual-polarized antennas. The system achieves higher spectrum efficiency with lower feedback overhead. The MIMO channel is decomposed into long-term components and short-term components [4]. Although the research perspective is forward-looking, there are still many unachievable parts of the technology.

\subsection{Innovation}

The innovations of this article are as follows: (1) Establish a supply chain network risk investigation framework model based on complex network theory. This paper takes supply chain network risk as a research difficulty and uses virus growth models, node importance evaluation models, immune system theory, and other methods to identify and evaluate supply chain risks and transmission mechanisms 
and systematically build immune strategies and network optimization design molds. This paper constructs a supply chain security evaluation model and indicator system. This indicator system classifies supplier evaluation indicators from different perspectives, which can reflect the performance of candidate suppliers in a more systematic and detailed manner and provide a reference for future research. (2) We propose a multi-index evaluation model based on the "best strategy" (best strategy, TOS). (3) Establish a multilevel evaluation model from business risk to supply chain network risk. (4) The SIS-RP diffusion model introduces the supply chain risk diffusion model. Supply chain risk communication research introduces dynamic communication theory in complex networks and introduces the SIS-RP dynamic risk communication model. This better reflects the macro-process of supply chain risk communication in the network.

\section{Complex network theory}

Supply chain risk management and complex network theory have been hot research topics in recent years. The core work of this article is to conduct cross-cutting research between complex network theory and supply chain risk and explore the research of complex network theory in supply chain risk management. Therefore, before carrying out various studies, it is necessary to comprehensively discuss the concepts, origins, and main research results of theories such as risk, supply chain, supply chain risk, and complex networks to provide basic theoretical support for subsequent research. A supplier evaluation index system model was established, the reasons for its selection based on the supply chain were explained, and the evaluation indices were processed in a dimensionless manner.

\subsection{Statistical characteristics of complex networks}

In recent years, people have proposed many concepts and methods for describing the topological structure of complex network structures. Among them, there are four basic statistical concepts: degree distribution, average path length, clustering coefficient (clustering coefficient), and betweenness. The statistical characteristics of these networks are introduced below $[5,6]$.

\section{(1) Degree and degree distribution}

The number of edges connected to a node (the number of neighboring nodes) is the degree of the node, which can be defined by the adjacency matrix [7]. $k_{i}=\sum_{j \in N} a_{i j}$

The object described by the degree index is a specific node in the network, which usually represents the influence of the node, and the greater the degree, the greater its influence. The node degree reflects only the nature of a single node. If all the node degrees in the network are summed and the arithmetic average is calculated, the average node degree of the network is obtained, which is generally denoted as $<k>$.

$\langle k\rangle=\frac{1}{N} \sum_{i=1}^{N} k_{i}$

Degree distribution refers to the probability that a node will be randomly selected by a network with degree $k$. This can be represented by the probability distribution function $P$, which represents the dispersion of each node in the network $[8,9]$. Statistically speaking, it can be expressed as the ratio of the number of nodes with degree $k$ to the total number of network nodes:

$P(k)=\frac{n_{k}}{N}$

where $n_{k}$ represents the number of nodes with node degree $k$, and $N$ represents the network scale, that is, the total number of nodes in the network [10]. Another index describing the statistical characteristics of network degrees is the cumulative degree distribution. As the name suggests, the cumulative degree distribution is the probability that the degree of a statistical node is greater than or equal to $k$, expressed as follows:

$P_{k}=\sum_{k_{1}=k}^{\infty} P\left(k_{1}\right)$

The average path length is a measure used to describe the effectiveness of network information or hardware forwarding and refers to the average shortest distance between all pairs of nodes in the network [11, 12]. The shortest distance between two nodes and the average path length of the $d_{i j}$ network are $N$, so the total number of edges that intersect the fastest path between any two nodes $i$ and $j$ in the network can be determined:

$L=\frac{2}{N(N-1)} \sum_{i \geq j} d_{\mathrm{ij}}$

$L$ is the path and $N$ is the number of nodes. $i, d, j$ are the corresponding parameters. Because the calculation process of the average path length is relatively complicated, it is often difficult to calculate it through the abovementioned method for a large-scale network [7]. After studying the WWW network, Albert et al. proposed the fitting 
relationship between the average shortest path length of a large-scale network and the network node size $N$, as shown below:

$L=0.35+2.06 \lg (N)$

This fitting relationship is mainly based on the WWW network. It is only an approximate algorithm and is not suitable for all large-scale networks.

\section{(2) Clustering coefficient}

Supplier evaluation and selection in the supply chain environment is different from the evaluation index system used in general supplier evaluation and selection. The general supplier evaluation index pays more attention to some short-term, procurement-related indicators, such as price, quality and delivery time, historical performance, etc. The clustering coefficient, also known as the cluster coefficient, is an important parameter that has attracted much attention in complex network theory and empirical research, and it describes the degree of clustering between nodes [13]. Assuming that the degree of the number of nodes in the network is $n_{i}$, the number of neighboring nodes of this node is $n_{i}$. Obviously, there can be at most $n_{i}$ edges between the $n_{i}\left(n_{i}-1\right) / 2$ neighboring nodes. Assuming the actual number of edges $E_{i}$ between the $n_{i}$ neighboring nodes, the clustering coefficient of node $i$ can be obtained as $C_{i}$ :

$C_{i}=\frac{2 E_{i}}{n_{i}\left(n_{i}-1\right)}$

In the network group based on the $5 \mathrm{G}$ information system, at the same time, it can be considered that the socalled supply chain refers to the supply and demand network composed of raw material suppliers, wholesalers, retailers, and final consumers involved in product production and circulation. The clustering coefficient can be used not only to measure the degree to which a node in a network and its neighboring nodes gather to form a community but also to calculate the clustering coefficient $C$ of the entire network by averaging the clustering coefficients of all nodes in the network. The expression is:

$C=\frac{1}{N} \sum_{i=1}^{N} C_{i}$

The average clustering coefficient of the network can also be obtained by another equivalent method:

$C=\frac{3 \times \Delta}{N_{\Delta}}$

where $\triangle$ is the total number of triangles in the network, and $M$ is the total number of three connected nodes in the network [14].

\section{(3) Betweenness}

Betweenness can reflect the comprehensive role and influence of nodes or edges in the entire network. Unlike node degree, betweenness is more focused on describing the global importance of nodes or edges, while node degree focuses on the importance of nodes at the micro-level [15]. Therefore, the expression formula for the betweenness of node $i$ is:

$g(i)=\sum_{s \neq t, s \neq v} \frac{\delta_{\mathrm{st}}(i)}{\delta_{\mathrm{st}}}$

where $\delta_{\mathrm{st}}(e)$ is the number of shortest paths between nodes passing through node $f$, and $\delta_{\text {st }}$ is the total number of all shortest paths between node $S$ and node $t$. Similarly, edge intermediary $(\mathrm{EB})$ is defined as the ratio of the shortest path through an edge to all the shortest paths calculated in the network [16]. Therefore, the expression for the betweenness of edge $e$ is:

$g(e)=\sum_{s \neq t, s \neq v} \frac{\delta_{\mathrm{st}}(e)}{\delta_{\mathrm{st}}}$

Among them, $\%(P)$ is the number of shortest paths between node $J$ and node $f$ through edge $e$.

\subsection{Classification of complex networks}

There are multiple classification methods for complex networks. For example, the network can be divided into directed and undirected networks, depending on the direction of the network edge. According to the weight of the edge in the network, it can be divided into weighted and unweighted networks and dense and sparse networks (depending on the edge) $[17,18]$. However, usually from the perspective of network topology, the main classification of complex networks can be roughly divided into regular networks, random networks, small-world networks, and scale-free networks (BAs). The following is a brief introduction to the network generation model and network characteristics.

The finally constructed 5G information system can efficiently operate the entire supply chain, reduce costs, improve services, and enhance rapid response capabilities, thereby providing practical support and guarantees for improving core competitiveness. A regular network is a network with a certain degree of regularity in the topological structure and translational symmetry. The closest adjacent link network is a network in which each node in the network can only be connected to the closest adjacent node $K / 2$ on both sides of the network, where $K$ is an even number less than or equal to IV, and the value of $K$ is directly related to the dilution of the network. Obviously, 
the nearest neighbor link network model is a network model with a ring structure and periodic boundary conditions $[19,20]$. If the value $K$ is large, the grouping factor $C$ of the adjacent link network model is:

$C=\frac{3(k-2)}{4(K-1)} \approx \frac{3}{4}$

For a fixed value of $K$, the average path length $L$ of the nearest neighbor coupling network is:

$L=\frac{N}{2 K} \rightarrow \infty$

The star coupling network has only one central node with the highest degree of nodes, and the remaining nodes are only connected to this central node [21, 22]. Therefore, for a star-connected network, the degree of the central node is $\mathrm{N}-1$, and the degree of other nodes is 1 . The clustering factor $C$ and the average path length 3 of the closest adjacent link model are:

$C=\frac{N-1}{N} \rightarrow 1(N \rightarrow \infty)$

$L=2-\frac{2(N-1)}{N(N-1)} \rightarrow 2(N \rightarrow \infty)$

\section{Supply chain information flow evaluation model}

With the issuance of China's 4G LTE license along with the help of new technologies, wireless networks based on cellular networks have once again improved the overall performance of the network. In addition, the popularity of smartphones, the rise of mobile internet, the emergence of new mobile applications, new services, new user experiences, and the demand for new mobile communication systems have led to a huge demand for wireless mobility. Strict delay requirements, not only in the network but also in time, have led to huge energy consumption. Today's latest $4 \mathrm{G}$ networks can no longer meet the future demand for high-speed data transmission and mobile communications. The industry has been studying fifth-generation mobile communication systems one after another. By analyzing the principles and processes of supplier evaluation index system construction, as well as existing research results at home and abroad, a supplier evaluation index system based on the supply chain was constructed. Figure 1 shows the $5 \mathrm{G}$ network architecture.

\subsection{Construction of risk assessment index system}

Combining the description of supply chain information flow risk and the analysis of the factors affecting the internal information flow risk of supply chain node enterprises in accordance with the existing principles and standards, through the analysis and combination of relevant documents, a preliminary supply chain enterprise internal information flow risk assessment index system is obtained. The internal information flow risk assessment indicator system of supply chain enterprises is composed of three secondary indicators and nine tertiary indicators. The specific indicator system is shown in Fig. 2:

Therefore, this study conducted interviews and questionnaire surveys with experts familiar with the content of this research at various universities in Yunnan Province and used a Likert scale to quantitatively analyze the impact of various indicators on the information flow risk of supply chain enterprises. To improve research efficiency and avoid duplication of work, the questionnaire was divided into two parts. At the same time, it investigated the indicators that affect the risk of internal information flow of supply chain node enterprises and the indicators that affect the risk of information flow between supply chain node enterprises. The impact of the indicator was divided into five levels: "huge impact", "large impact," "normal impact," "small impact," and "basically no impact," and values of 5, 4, 3, and 2 were assigned in turn. 1. A total of 110 questionnaires were distributed in this survey, and 84 questionnaires were recovered, with an effective rate of $76.36 \%$. First, the reliability of the questionnaire was analyzed, and the Cronbach coefficient was used for reliability measurement. The data collected in this questionnaire were analyzed by SPSS 17.0 software, and the results obtained are shown in Table 1:

Table 1 shows that the total reliability index of the questionnaire was Cronbach $=0.825>0.8$, indicating that the reliability of the questionnaire survey was relatively high. Subsequently, SPSS 17.0 software was used to perform factor analysis to screen the indicators. $F 1 \sim F 9$ in turn represent information processing capability, corporate information management system, internal network security, corporate organizational structure, information uncertainty, ideology, degree of informatization, information management personnel quality, and employee information security awareness. The principal component analysis method was used, the maximum variance orthogonal rotation method was used to rotate, and the number of factors extracted according to the principle that the eigenvalue was greater than 1 . The score coefficient matrix of the factor analysis results is shown in Table 2: 
Fig. $15 \mathrm{G}$ network architecture diagram

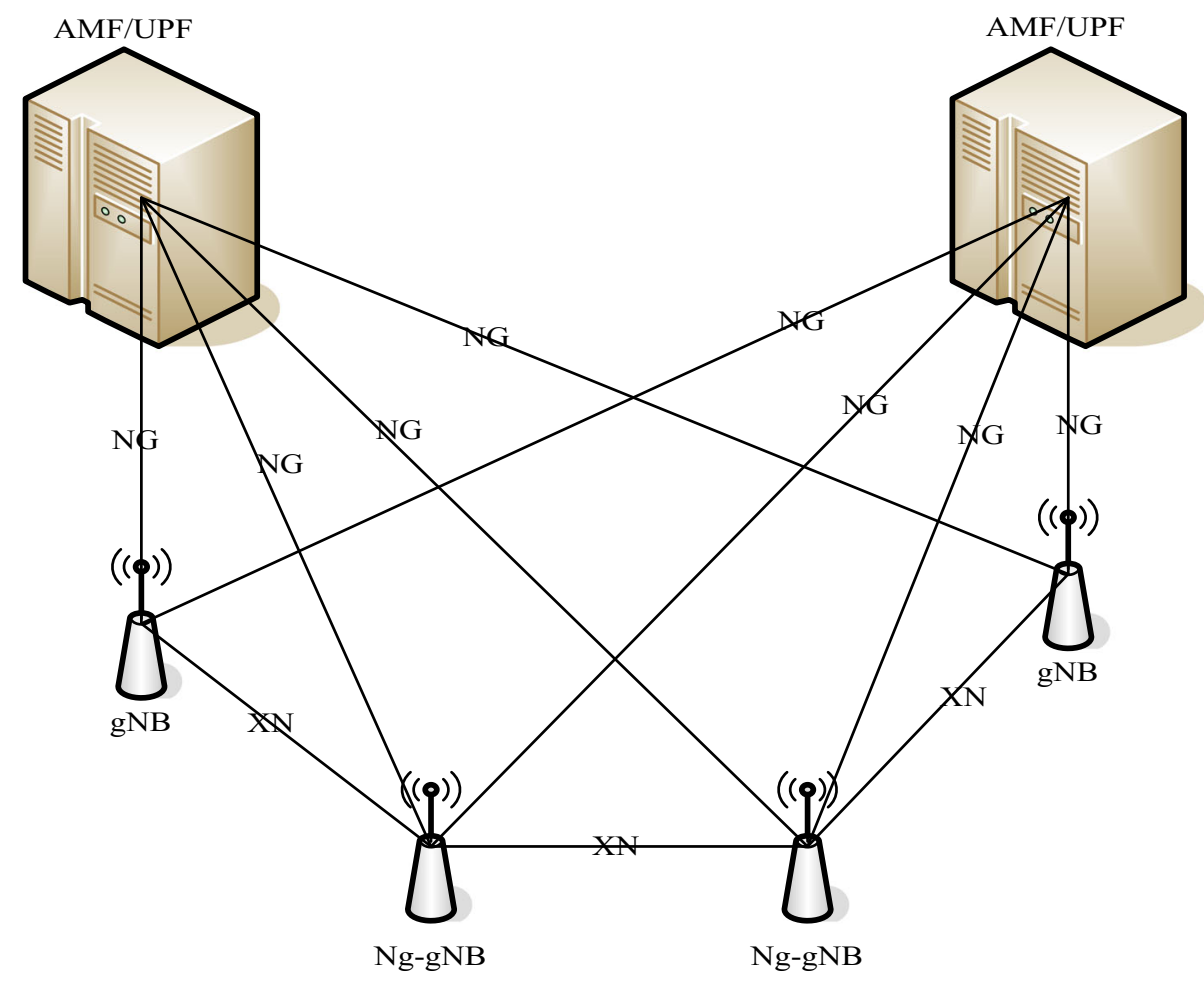

Supply chain information flow risk assessment index system

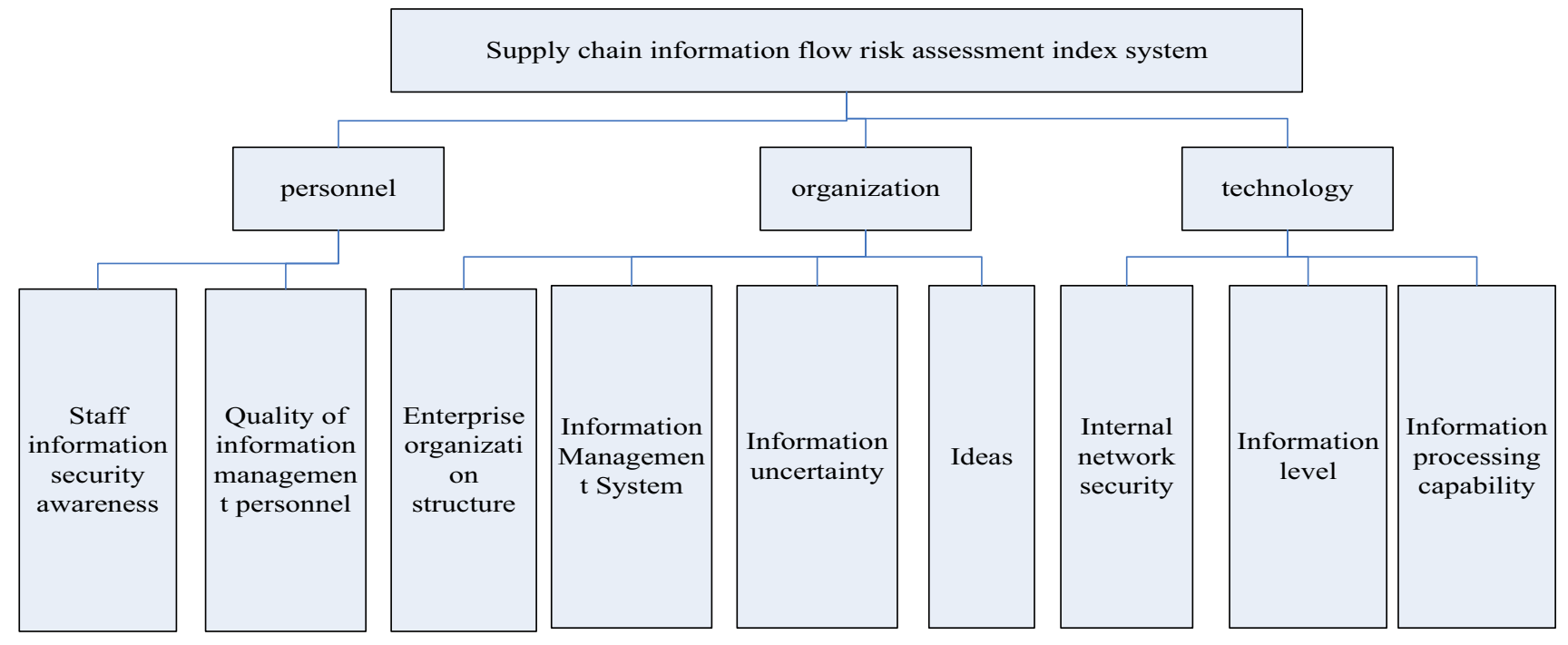

Fig. 2 Preliminary indicator system of supply chain information flow risk assessment

Table 1 Total reliability statistics table

\begin{tabular}{lll}
\hline Cronbach's alpha & Cronbach's alpha based on standardized items & $N$ of items \\
\hline 0.825 & 0.822 & 9 \\
\hline
\end{tabular}

The supplier evaluation indicator system in the supply chain environment pays more attention to indicators related to strategy, sustainability, complementarity, and compatibility. According to the data in Table 3, the indices whose loads in all factors are all less than 0.5 are eliminated. That is, the indicators "corporate organizational structure," "information uncertainty of oneself," "degree of informatization," and "employee information security awareness" are removed from the evaluation system. 
Table 2 Factor scoring coefficient matrix

\begin{tabular}{lrrrrr}
\hline$W^{(0)}$ & \multicolumn{6}{c}{ Component } & & & \\
\cline { 2 - 6 }$W^{(1)}$ & 1 & \multicolumn{2}{l}{3} & \multicolumn{1}{l}{5} \\
\hline$F 1$ & 0.506 & 0.054 & -0.264 & 0.040 & 0.332 \\
$F 2$ & -0.032 & -0.102 & 0.036 & 0.782 & -0.017 \\
$F 3$ & -0.052 & 0.618 & 0.005 & -0.228 & -0.039 \\
$F 4$ & 0.456 & -0.195 & -0.003 & 0.008 & -0.112 \\
$F 5$ & 0.214 & -0.096 & 0.456 & -0.377 & 0.006 \\
$F 6$ & 0.004 & 0.008 & 0.076 & -0.037 & 0.780 \\
$F 7$ & -0.332 & -0.325 & -0.121 & 0.011 & 0.364 \\
$F 8$ & -0.214 & 0.040 & 0.743 & 0.195 & -0.006 \\
\hline
\end{tabular}

Table 3 Combination weight table of subjective and objective matrix

\begin{tabular}{lllll}
\hline & $B 1$ & $B 2$ & $B 3$ & $W$ \\
& 0.338 & 0.5906 & 0.0711 & \\
\hline$P 1$ & 0.0958 & 0.3952 & 0.3311 & 0.2945 \\
$P 2$ & 0.1157 & 0.1845 & 0.2358 & 0.1658 \\
$P 3$ & 0.1821 & 0.1358 & 0.2306 & 0.1595 \\
$P 4$ & 0.2445 & 0.1985 & 0.1375 & 0.2056 \\
$P 5$ & 0.3625 & 0.0458 & 0.0741 & 0.1787 \\
\hline
\end{tabular}

\subsection{Combining rough set theory and analytic hierarchy process to construct objective matrix}

On this basis, an objective judgment aid is constructed, using $p 1, p 2, p 3, p 4$, and $p 5$ as the condition attributes, and $d$ represents the decision attribute, corresponding to the decision attribute $D$ (criterion layer). Eleven experts were selected to evaluate the importance of the correlation between "risk probability," "risk impact," and "uncontrollability" and the five security events at the program level. According to the difference in the importance of their correlation, the value range is $\{1,2,3,4\}$, and the meaning of the attribute value represents 1 (no impact), 2 (slight impact), 3 (general impact), and 4 (very impact). The following selects 11 experts as the object set $U$. The experts evaluated the security incidents based on their own experience and experience. According to the combined weight calculation formula $W=W(1) W(2)$ of each security incident regarding the importance of the security incident, Table 3 is obtained, and the subjective and objective matrix combination weight table is shown.

The results show that the final combined weights of risk factors $P 1, P 2, P 3, P 4$, and $P 5$ are $0.2945,0.1658,0.1595$, 0.2056 , and 0.1787 , respectively. The security incident "hardware failure" $(P 1)$ has the highest degree of risk, followed by "data leakage" $(P 4)$, followed by "denial of service" (P5), and "operational error" $(P 2)$. The lowest security risk is "Denial of Service" $(P 3)$. Since $P 1$ has a relatively large security risk weight, when performing security control on the information system, the measures that should be taken are regular and high-frequency inspections of computer hardware.

\subsection{Hybrid nonlinear goal programming model}

One relevant example is the cigarette supply and marketing network of a province in China. Because cigarettes are monopoly products prescribed by the state, a provincial tobacco company is responsible for the overall planning and implementation management of the entire supply and marketing network. At present, there are three cigarette production enterprises (manufacturers) in the province, and the production capacity of each manufacturer is cap = 2000. To further expand the market, the provincial headquarters promoted a new product plan and planned three alternative distribution centers and nine alternative sales markets around the distribution and sales of new products. Therefore, the cigarette supply and marketing network is a three-level supply chain network. The following is a simulation analysis of the optimization design problem of the distribution center and sales market layout in the cigarette supply chain network, as shown in Table 4:

As the probability of Condition 2 increases, the probability of Condition 1 and Condition 4 decreases and increases. The level of profit is declining significantly. At the same time, due to the increase in the possibility of interruption by manufacturer No. 2, the supply chain path with manufacturer No. 2 as the origin no longer occupies a major position, and the No. 2 alternative distribution center through which these supply chain paths pass is naturally taken from. The supply chain network was removed, which also resulted in the removal of the No. 4 and No. 5 sales markets that only received products from the No. 2 distribution center from the supply chain network. However, this layout changed when the probability of situation 2 increased to 0.55 . This is mainly because the supply of the entire product becomes increasingly secure when the possibility of interruption of the No. 2 manufacturer reaches a certain level. At this time, the product supply will be biased toward the market with more demand and greater profit. Due to the high cost of the supply chain path through the No. 1 alternative distribution center and low market demand, the No. 2 alternative distribution center has obvious advantages. Therefore, the No. 1 alternative distribution center was eliminated, and the No. 2 alternative distribution center was reincorporated into the supply chain network. At the same time, alternative sales market No. 
Table 4 Sensitivity analysis results of the probability change of the interruption status

\begin{tabular}{|c|c|c|c|c|c|c|}
\hline & Situation 1 probability & Situation 1 probability & Situation 1 probability & Situation 1 probability & Profit & Layout point \\
\hline 1 & 0.825 & 0.05 & 0.1 & 0.005 & 2042 & $1 / 3$ \\
\hline 2 & 0.751 & 0.15 & 0.1 & 0.015 & 1782 & $1 / 3$ \\
\hline 3 & 0.632 & 0.25 & 0.1 & 0.025 & 1558 & $1 / 3$ \\
\hline 4 & 0.515 & 0.35 & 0.1 & 0.035 & 1312 & $1 / 3$ \\
\hline 5 & 0.412 & 0.45 & 0.1 & 0.045 & 1066 & $1 / 3$ \\
\hline 6 & 0.268 & 0.55 & 0.1 & 0.055 & 882 & $2 / 3$ \\
\hline 7 & 0.185 & 0.65 & 0.1 & 0.065 & 802 & $2 / 3$ \\
\hline
\end{tabular}

1.2.3, which only picked up goods from alternative distribution center No. 1, was also eliminated. It can also be found that, compared with the No. 1 manufacturer, the supply chain path with the No. 2 manufacturer as the point of origin has greater profit margins.

This section mainly considers the problem of supply chain network optimization design when market demand is uncertain and product supply is interrupted due to risk events. A hybrid nonlinear objective programming model is given, and the model is analyzed from the perspective of the robustness of the solution: Optimized around the problem of solving the model, a method based on piecewise linear transformation is proposed, and finally, a case was used to model and solve the target programming problem, which verifies the effectiveness of the model and the solution method.

\section{Experiment and result analysis}

This paper analyzes the experimental data and verifies the validity and accuracy of the acceptability evaluation model through simulation experiments. The direction of the experiment is mainly to verify the validity of the trust model and the risk model in the acceptability model, as well as the comparison between the acceptability model and the Josang evaluation model.

The feasibility verification of the e-commerce network transaction model needs to consider the following three parts: (1) the validity verification of the trust degree model; (2) the validity verification of the risk degree model; and (3) the validity verification of the acceptability model feasibility verification. Because the global reputation is only the expected value of the local reputation of the node relative to the neighbor node in the experiment of the simulation trust degree model, it is only necessary to verify the accuracy of the local reputation calculation and the reliability of the trust degree model; in the experiment of the risk degree model simulation model, verify the influence of risk factors on the results of risk calculation; in the simulation experiment of the acceptability model, the simulation compares the acceptability model and the Josang model. To accurately ensure the validity and credibility of the experimental results, representative data are selected for simulation experiments to improve the reliability of the experimental results.

Experiment 1: Validity analysis of acceptability model.

Figure 3 shows that as the transaction time is farther away from the current moment, the local reputation gradually decays and converges to a fixed value. From the figure, it can be found that if the transaction amount between nodes is larger, the node evaluation accounts for the greater the proportion of, the greater the local reputation value of the node.

In addition to factors such as cost, quality, and delivery to investigate and study the evaluation index system for companies choosing suppliers, researchers must also consider the relationship of creditworthiness. Figure 4 shows the change value of the node's trust degree in a certain period of time. It can be seen from the figure that when the global reputation value is 0.5 and the local reputation value is 0.8 and 0.7 , the trust degree changes with the change of the confidence factor. It can be seen that as the value of the confidence factor becomes smaller and smaller, the trust degree converges more and more to the global reputation, and vice versa.

Figure 5 simulates the impact of risk factors and transaction failure values on the results of the risk calculation. With the continuous delay of online transaction time, the failure value of the transaction gradually increases. It can be seen from Fig. 5 that when the value of the risk factor is $0.4,0.7$, and 0.8 , the calculation result of the risk degree will gradually become larger and eventually converge toward a certain fixed value: When the failure value of the network transaction gradually increases, the risk of online transactions has also become greater.

Experiment 2: Comparative experiment. 
Fig. 3 The impact of transaction time and transaction amount on the node's local reputation

Fig. 4 The influence of confidence factor on trust

Fig. 5 The impact of risk factors and transaction failure values on risk
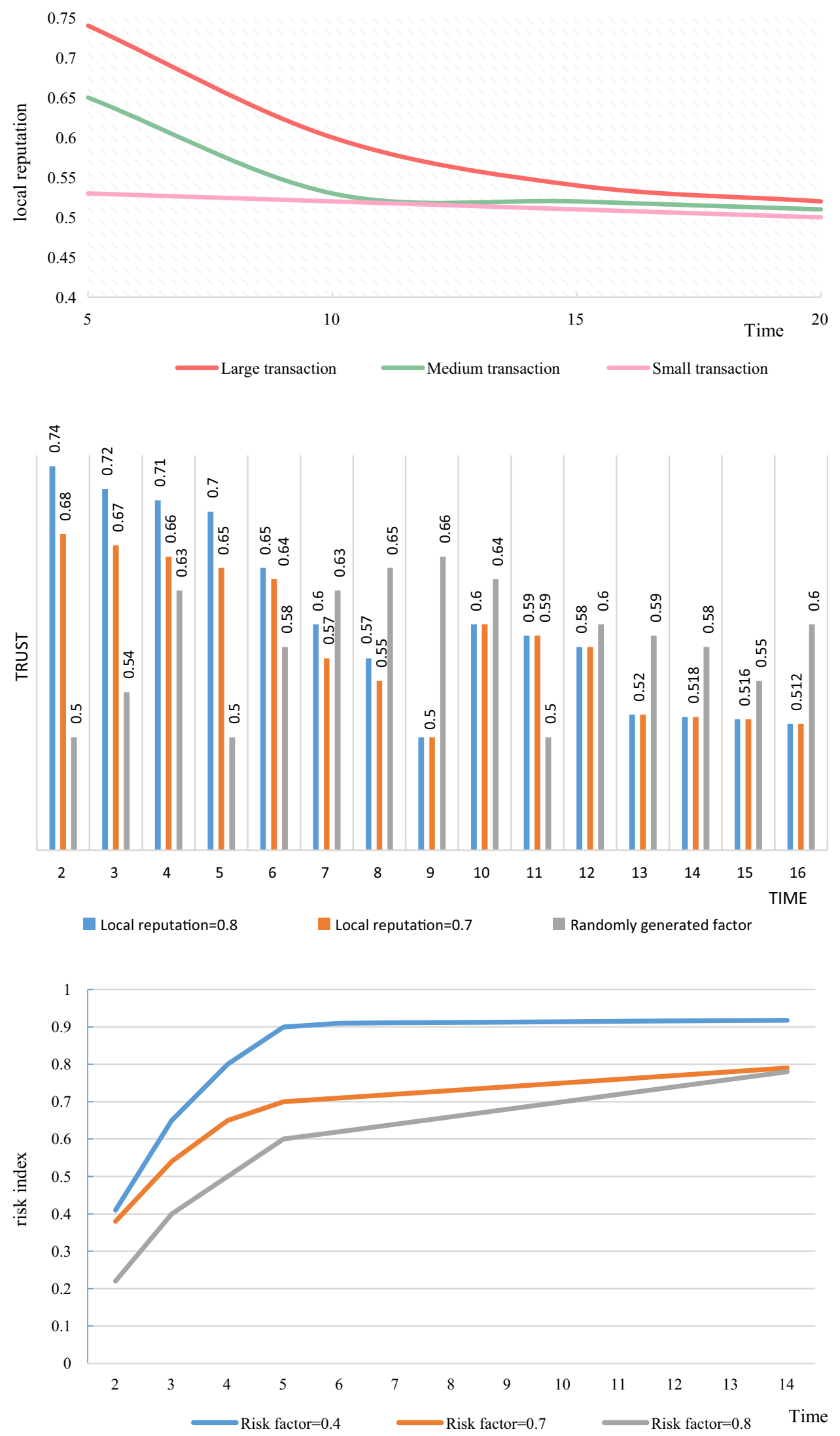
Fig. 6 Comparison of the acceptability model and Josang reputation model
0.8

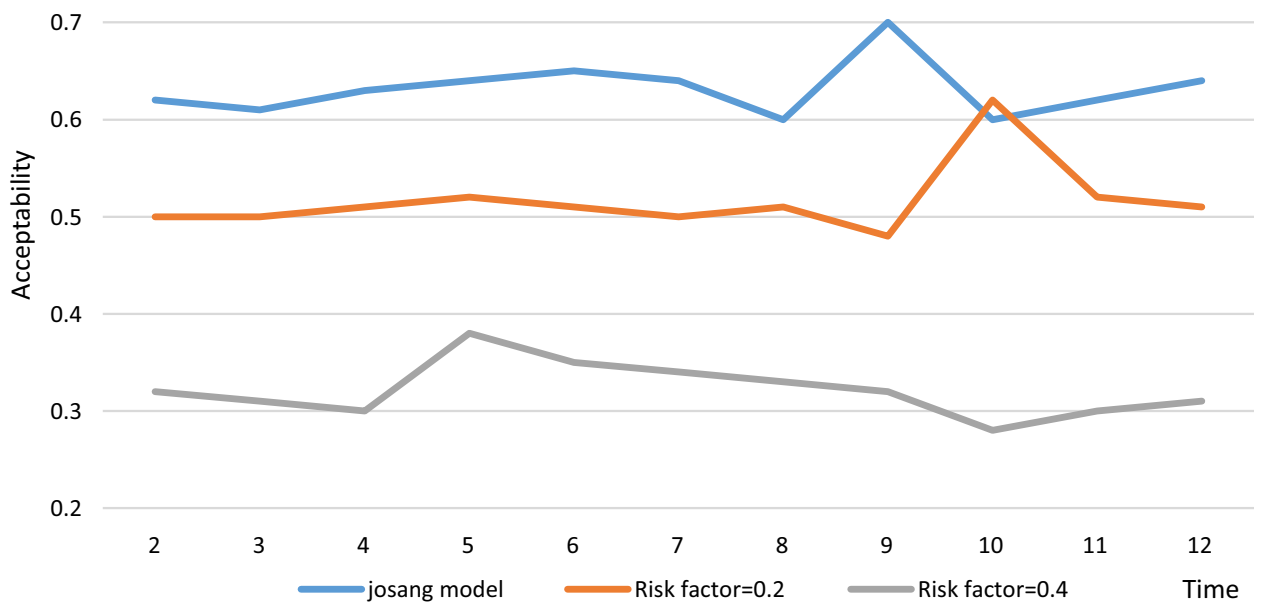

The simulation in Fig. 6 compares the acceptability model and the Josang reputation model. It can be seen from Fig. 6 that the acceptability model comprehensively considers trust and risk, and the calculated value is lower than Josang and other reputation-based evaluation models, which can effectively avoid transactions with malicious nodes. Therefore, when the risk degree is introduced into the transaction evaluation model, the node transaction decision result of the model evaluation is more accurate and reliable.

Experimental conclusion: The acceptability transaction evaluation model proposed in this paper combines objective risks into transaction decisions for the first time, making the results of model calculations more accurate, effectively improving the accuracy of nodes participating in network transaction decisions, and thus avoiding inaccurate transactions occurring at the node.

\section{Conclusion}

To improve agility, many companies invest much money to build cross-organizational information systems to enhance coordination between companies so that they can quickly respond to continuous market changes. However, many companies cannot adapt well to changing demands. With the advent of the internet and the era of big data, the business environment has become increasingly complex. To adapt to changes in the environment, traditional supply chain companies have evolved into a complex system with increasingly larger scales and increasingly complex organizations through continuous development. In the process of this change, faced with its own large scale and complex organization, the supply chain risk problem that the company has to solve has become increasingly prominent. In the production and operation of supply chain enterprises, information flow is an important channel connecting logistics and capital flow. With the increase in supply chain business partners, the growth of the number of transactions, and the diversification of cooperative relations, the data that enterprises need to receive and output are increasing. In the face of significant growth in the amount of information, it is particularly important for companies to guide the correct, efficient, and safe flow of information. The ever-increasing information flow is filled with considerable information noise, and only good information flow management can provide decision support for the enterprise's logistics and capital flow; otherwise, it will only mislead managers to make incorrect decisions. Therefore, if companies intend to develop well and stand out in today's highly competitive environment, they must pay attention to the risk of information flow in the supply chain. Compared with the traditional supply chain, the 5G information system brings more benefits to enterprises. For example, flexibility and rapid market response enable enterprises to obtain higher financial performance. The reason behind the competitive advantage brought by these technologies in the supply chain is the enhancement of the perception and resilience enabled by information technology. In addition to directly improving the two agility capabilities of the enterprise, the flexible $5 \mathrm{G}$ information system in this article can also alleviate the negative impact of supply chain resource advantages on agility.

\section{Declarations}

Conflicts of interest The authors declare that there is no conflict of interest with any financial organizations regarding the material reported in this manuscript. 


\section{References}

1. Weber R (2018) Cyber threat from China unifies congress on setting tough security protections. Inside Pentagon 34(51):11-12

2. Supply CB (2017) Supply chain security. Aviat Week \& Space Technol 179(22):12-12

3. Barbosa MW (2021) Uncovering research streams on agri-food supply chain management. Glob Food Secur 28(1):100517

4. Modarress B, Ansari A, Thies E (2016) Outsourcing in the Persian Gulf petroleum supply chain. Strateg Outsourc Int J 9(1):2-21

5. Feng Z, Chen Y, Qian Z et al (2017) An efficient CSI feedback scheme for dual-polarized MIMO systems using layered multipaths information. China Commun 14(5):91-104

6. Lu G, Koufteros X, Lucianetti L (2017) Supply chain security: a classification of practices and an empirical study of differential effects and complementarity. IEEE Trans Eng Manag 64(2):234-248

7. Ni JZ, Melnyk SA, Ritchie WJ et al (2016) Why be first if it doesn't pay? The case of early adopters of C-TPAT supply chain security certification. Int J Oper Prod Manag 36(10):1161-1181

8. Forte D, Perez R, Kim Y et al (2016) Supply-chain security for cyberinfrastructure [Guest editors' introduction]. Computer 49(8):12-16

9. Tabor J (2016) No silver bullet for supply chain security. Am Shipp 58(9):46-46

10. Zhu Y, Kumar S, Rodriguez-Sanchez S et al (2016) Managing logistics in regional banknote supply chain under security concerns. Prod Oper Manag 24(12):1966-1983

11. Lu G, Koufteros X (2019) Organizing practices to combat supply chain security breaches. IEEE Eng Manag Rev 47(3):72-78

12. Mehta Dhyey, Tanwar Sudeep, Bodkhe Umesh, Shukla Arpit, Kumar Neeraj (2021) Blockchain-based royalty contract transactions scheme for Industry 4.0 supply-chain management. Inf Process Manag 58(4):102586
13. Bertuca $T$ (2019) Trump issues new executive order on supply chain security. Inside Pentagon 35(21):3-3

14. Doubledy J (2019) Senators press prospective DepSecDef on cyber, supply chain security. Inside Navy 32(30):3-4

15. Justin D (2018) DOD considers tax incentives to strengthen supply chain security. Inside Pentagon 34(3):1-12

16. Whitfield B (2018) Attack path discovery: supply chain security. Port Technol int 79:100-101

17. Everett S, Pratama DH (2017) Supply chain security initiatives: the authorized economic operator and indonesia's experience. J Int Logist Trade 15(1):8-16

18. Leng K, Jing L, Lin IC et al (2019) Research on mining collaborative behaviour patterns of dynamic supply chain network from the perspective of big data. Neural Comput \& Appl $31: 113-121$

19. Eric K (2016) Co-op approach to supply chain security. Am Shipp 58(12):25-27

20. Sun L, Zhao Y, Sun W et al (2020) Study on supply chain strategy based on cost income model and multi-access edge computing under the background of the Internet of Things. Neural Comput \& Applic 32:15357-15368

21. Bader Lennart, Pennekamp Jan, Matzutt Roman, Hedderich David, Kowalski Markus, Lücken Volker, Wehrle Klaus (2021) Blockchain-based privacy preservation for supply chains supporting lightweight multi-hop information accountability. Inf Process Manag 58(3):102529

22. Santiba Ez-Aguilar JE, Guillen-Gosálbez G, Morales-Rodriguez $R$ et al (2016) Financial risk assessment and optimal planning of biofuels supply chains under uncertainty. Bioenergy Res 9(4):1-17

Publisher's Note Springer Nature remains neutral with regard to jurisdictional claims in published maps and institutional affiliations. 